\title{
EZH2 Overexpression as a Useful Prognostic Marker for Aggressive Behaviour in Thyroid Cancer
}

\author{
KATSUHIKO MASUDO $^{1 *}$, NOBUYASU SUGANUMA ${ }^{1,2^{*}}$, HIROTAKA NAKAYAMA ${ }^{1}$, \\ TAKASHI OSHIMA ${ }^{1}$, YASUSHI RINO ${ }^{1}$, HIROYUKI IWASAKI ${ }^{2}$, KENICHI MATSUZU ${ }^{3}$, \\ KIMINORI SUGINO $^{3}$, KOICHI ITO ${ }^{3}$, TETSUO KONDO ${ }^{4}$, YOSHIYASU NAKAMURA ${ }^{5}$, \\ MITSUYO YOSHIHARA ${ }^{5}$, MUNETAKA MASUDA $^{1}$ and YOHEI MIYAGI ${ }^{5}$ \\ ${ }^{1}$ Department of Surgery, Yokohama City University, Yokohama, Japan; \\ ${ }^{2}$ Department of Breast and Endocrine Surgery, Kanagawa Cancer Center, Yokohama, Japan; \\ ${ }^{3}$ Department of Surgery, Ito Hospital, Tokyo, Japan; \\ ${ }^{4}$ Department of Human Pathology, University of Yamanashi, Yamanashi, Japan; \\ ${ }^{5}$ Molecular Pathology and Genetics Division, Kanagawa Cancer Center Research Institute, Yokohama, Japan
}

\begin{abstract}
Background/Aim: Enhancer of zeste homolog 2 (EZH2) is a member of the polycomb group of genes, which are key factors in the regulation of cell proliferation and differentiation. EZH2 is overexpressed in many malignancies. We analyzed EZH2 protein expression levels in different histological subtypes of thyroid cancer to examine its utility as a prognostic factor. Materials and Methods: We examined EZH2 protein expression by immunohistochemistry in tissue samples from 67 cases of poorly differentiated (PDTC) and 48 cases of anaplastic thyroid carcinoma (ATC), and in samples of adjacent normal and differentiated thyroid carcinoma (DTC). We examined differences in expression of EZH2 among various histological types of thyroid cancer, and the relationship between EZH2 expression and patient outcome. Results: EZH2 protein was expressed in PDTC and ATC, but not in normal thyroid gland or DTC. EZH-positivity increased in the order of DTC, PDTC, and ATC $(p<0.01)$. Higher EZH2 expression correlated with poorer survival in PDTC $(p=0.004)$, and a similar but non-significant trend was observed in ATC ( $p=0.166)$. Multivariate analysis identified EZH2 as an independent prognostic factor similar to metastatic status in the Japanese Society of Thyroid Surgery
\end{abstract}

This article is freely accessible online.

*These Authors contributed equally to this study.

Correspondence to: Nobuyasu Suganuma, Department of Surgery, Yokohama City University, 3-9 Fukuura, Kanazawa-ku, Yokohama 236-0004, Japan. Tel: +81 457872800, e-mail: n-suga@ vesta.dti.ne.jp

Key Words: Thyroid cancer, EZH2, prognostic factor, epigenetics.
(JSTS) classification of PDTC. Conclusion: EZH2 overexpression is associated with malignant potential in thyroid cancer, and may thus be a useful prognostic marker of aggressive thyroid cancer.

Thyroid cancer is the most common malignancy of the endocrine system, with an apparently increasing incidence associated with the widespread use of imaging studies $(1,2)$. The lifetime risk of thyroid cancer is approximately $1.1 \%$, but the 5-year survival rate has risen to $97.8 \%$ because almost $70 \%$ of cases are now diagnosed at an early stage while the cancer remains localized to the thyroid gland (3). There are five main histological types of thyroid carcinoma: papillary (PTC), follicular (FTC), medullary, poorlydifferentiated (PDTC), and anaplastic thyroid carcinoma (ATC) (4). PTC and FTC are the most common types of thyroid cancer; these are considered as well-differentiated thyroid carcinomas (DTCs) and have a very good prognosis. In contrast, ATC is relatively rare; it accounts for only 1-2\% of all thyroid cancers and has an extremely poor prognosis (5), with 6-month and 1-year cause-specific survival rates for common-type ATC of $36 \%$ and $18 \%$ (6). PDTC is a malignant tumor showing intermediate traits between DTC and ATC. Three types of PDTC have been defined by the Japanese Society of Thyroid Surgery (JSTS) criteria, World Health Organization (WHO) classification, and Turin proposal. The 6th edition of the General Rules for the Description of Thyroid Cancer by the JSTS (7) defined PDTC as a tumor with only a small poorly differentiated compartment, showing insular, trabecular, solid, or scirrhous growth patterns, based on the proposal by Sakamoto et al. (8). The WHO definition of PTCD (4) is a tumor with insular, trabecular, or solid patterns in most of the tumor, together with an infiltrative pattern of growth, necrosis, and 
obvious vascular invasion. The Turin diagnostic criteria for PTCD include the presence of insular/trabecular/solid growth pattern, absence of conventional nuclear features of papillary carcinoma, and the presence of at least one of convoluted nuclei, mitotic activity $\geq 3 \times 10 /$ high-power field, or tumor necrosis (9). The prevalence decreases and the prognosis worsens in the order JSTS, WHO, Turin (10).

Enhancer of zeste homolog 2 (EZH2) is a well-known histone modifier protein that functions as a methyltransferase at lysine 27 of histone $\mathrm{H} 3$ (11). EZH2 is a member of the polycomb group of genes (12), which is important for transcriptional regulation through chromatin remodelling, nucleosome modification, and interactions with other transcription factors. EZH2 is presumed to promote cancer progression by transcriptional repression of tumour suppressors and by maintaining cells in a stem-cell-like state $(13,14)$. EZH2 has been shown to be overexpressed in many types of malignancies, including prostate, bladder, breast, lung, gastric, and brain cancers and has been suggested as a candidate prognostic factor and therapeutic target $(15,16)$.

In thyroid cancer, high EZH2 expression levels have been detected in ATC, with no expression in normal thyroid and low expression in DTC (17). More aggressive disease, such as the occurrence of metastases in medullary thyroid carcinoma, was associated with significantly increased $E Z H 2$ gene expression, which did not correlate with the mutational statuses of the RET or RAS genes (18). However, there is currently no information on the expression of EZH2 in PDTC or on the relationship between EZH2 expression and prognosis in the different pathological types of PDTC.

This study aimed to analyze the expression levels of EZH2 protein in different histological types of thyroid cancer, correlate these expression levels with various clinicopathological factors, and examine the utility of EZH2 as a prognostic factor in patients with thyroid cancer.

\section{Materials and Methods}

Patients and samples. We retrospectively examined surgical specimens of thyroid tumors from patients who underwent surgery at Kanagawa Cancer Centre and Ito Hospital between December 1977 and March 2013. EZH2 expression was examined by immunohistochemistry in tissue samples from 67 cases of PDTC (defined by the JSTS 6th edition), 48 cases of ATC, and in 30 samples of adjacent normal and differentiated thyroid carcinoma tissue which come from some of the above PDTC/ATC patients.

The study protocol was approved by the Ethics Committees of the Kanagawa Cancer Centre and Ito Hospital.

Tissue microarrays. Archival hematoxylin and eosin-stained slides of the samples were retrieved and reviewed to confirm the pathological features and select suitable tissue blocks for immunohistochemistry analysis. We constructed tissue microarrays (TMAs) using PDTC, ATC, and the adjacent normal and DTC lesions. TMAs consisting of cores, each measuring $4 \mathrm{~mm}$ in diameter, were assembled from formalin-fixed, paraffin-embedded blocks of surgical samples of primary thyroid tumors. The array included tissue cores from 68 PDTC, 48 ATC, and 30 adjacent normal and DTC tissues as a control.

Immunohistochemistry. Immunohistochemical staining for EZH2 was performed in all cases. TMAs were cut into 4 - $\mu$ m-thick sections and mounted on pre-coated glass slides. All sections were stained with primary antibodies to EZH2 (clone D2C9, dilution 1:50; Cell Signaling Technology Inc., Danvers, MA, USA) using an autostainer (Histostainer; Nichirei Biosciences Inc., Tokyo, Japan) (19).

Immunohistochemical scoring was based on semi-quantitative assessment of the intensity of nuclear staining (graded as 0 , no staining; $1+$, weak; $2+$, medium; and $3+$, strong) and the percentage of positive cells. Positive staining was defined by strong staining $(2+$ or $3+$ ) and a high percentage positivity $\geq 10 \%$ (Figure 1 ).

The immunohistochemistry results were assessed in a blinded fashion by pathologists (Y.M. and T.K.) and a thyroid surgeon (N.S.) who examined each slide independently. Unclear cases were discussed between the pathologists and thyroid surgeon.

Follow-up. Follow-up data were obtained from the Kanagawa Cancer Centre and Ito Hospital registries until December 31, 2016. Active follow-up was conducted by accessing hospital-visit records, resident registration cards, and permanent-domicile data. During the study period, four subjects $(3.4 \%$; $4 / 115)$ were lost to follow-up. Overall survival (OS) was defined as the period from the day of diagnosis of the primary lesion until the day of death. The median follow-up time was 39 months [PDTC; 59 (range=8-122 months) months, ATC; 4 (range=0-151 months) months] after diagnosis of the primary lesion.

Statistical analyses. The comparison of patient characteristics among each pathological subtype was used Mann-Whitney $U$-test. Positive rates of EZH2 expressions in normal and neoplastic thyroid tissues, and associations between clinicopathological factors and EZH2 expression were analysed by chi-square test. OS was analysed using the Kaplan-Meier method, and Cox proportional hazards models were applied to the multivariate analyses. Correlations between EZH2 expression and clinicopathological factors were evaluated using Pearson's product-moment correlation coefficients (r). A $p$-value $<0.05$ was considered statistically significant for all statistical analysis. All statistical analyses were performed using SPSS version 20 (SPSS Inc., Chicago, IL, USA).

Ethical approval. All procedures performed in studies involving human participants were performed in accordance with the ethical standards of the institutional and/or national research committee and with the 1964 Helsinki declaration and its later amendments or comparable ethical standards.

Informed consent. Informed and signed consent was obtained from all individual participants included in the study.

\section{Results}

Clinicopathological characteristics of all patients. The tissue samples were obtained from 67 cases of JSTS, 37 cases of WHO and 12 cases of Turin PDTC, 48 cases of ATC, and in 30 samples of adjacent normal and differentiated thyroid 


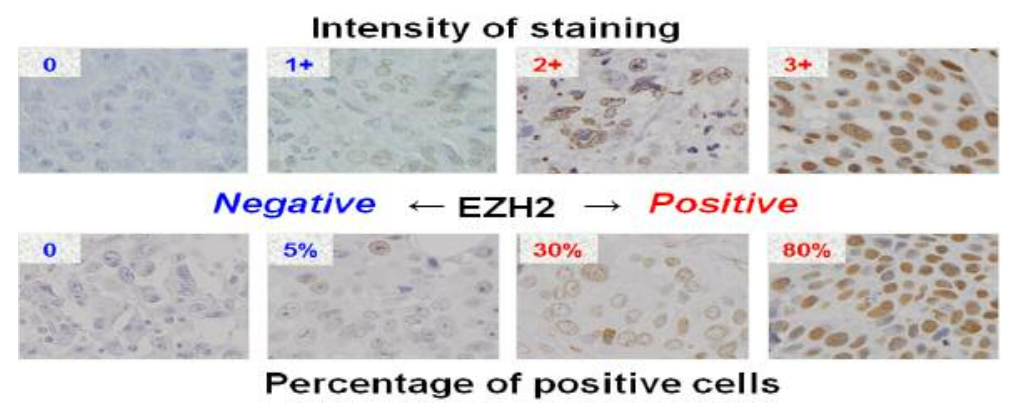

Figure 1. Representative breast tissue sections stained with EZH2 antibody. Immunohistochemical scoring was done by semi-quantitative assessment of the intensity of nuclear staining (graded as 0 , no staining; $1+$, weak; $2+$, median; and $3+$, strong) and the percentage of positive cells. Positive staining was defined by strong staining $(2+$ or $3+)$ and high percentage positivity $\geq 10 \%$.

carcinoma tissue which come from some of the above PDTC/ATC patients. The patient characteristics are summarized in Table I. The mean ages of the study subjects were 55 years (range $=19-83$ years) for JSTS, 53 years (range $=9-83$ years) for $\mathrm{WHO}$, and 58 years (range $=29-72$ years) for Turin PDTCs, and 68 years (range $=48-90$ years) for ATC. There were significant differences in age between PDTC and ATC (JSTS $v s$. ATC/WHO vs. ATC/Turin $v s$. ATC $=p<0.001 / p<0.001 / p=0.033$, respectively), but no significant differences among the three pathological types of PDTC. There were no significant differences between any of the four groups (JSTS, WHO, Turin, and ATC) in terms of sex or M status.

EZH2 status in each pathological type. EZH2 expression was evaluated by immunohistochemistry using paraffinembedded sections of normal and carcinoma thyroid tissues. Representative images and the EZH2-positivity of each pathological type are shown in Figure 2. EZH2 protein expression was detected in $87.5 \%(42 / 48)$ of ATC cases, but in no normal thyroid or DTC samples. EZH2 protein expression was detected in $17.9 \%$ (12/67) of JSTS, $21.6 \%$ $(8 / 37)$ of WHO, and $25.0 \%$ (3/12) of Turin PDTCs, with a significant increase in the order DTC $<$ PDTC $<$ ATC $(p<0.01)$.

Associations between clinicopathological factors and EZH2 expression. The associations between clinicopathological factors and EZH2 expression are shown in Table II. EZH2 showed no significant association with any factors in JSTS and Turin criteria PDTC or ATC, but high expression of EZH2 was associated with smaller tumor diameter in WHO classification PDTC. There was no significant difference in EZH2 expression or T status between JSTS and Turin criteria PDTC.

Relationship between EZH2 expression and patient outcome. Univariate analysis showed that high EZH2 expression was a poor prognostic indicator of OS in JSTS classification
Table I. Patient characteristics

\begin{tabular}{llccc}
\hline & & \multicolumn{3}{c}{ PDTC } \\
\cline { 3 - 5 } & & JSTS (n=67) & WHO (n=37) & Turin (n=12) \\
\hline Age (yr) & $\geq 55 /<55$ & $38 / 29$ & $18 / 19$ & $09 / 03$ \\
Gender & male/female & $26 / 41$ & $14 / 23$ & $03 / 09$ \\
T (cm) & $\geq 4 /<4$ & $44 / 23$ & $29 / 08$ & $09 / 03$ \\
Ex & Ex2/Ex0, & $02 / 65$ & $01 / 36$ & $00 / 12$ \\
N & N1b/N0, 1a & $08 / 59$ & $01 / 36$ & $01 / 11$ \\
M & M1/M0 & $17 / 50$ & $08 / 29$ & $04 / 08$ \\
& & & & \\
& & ATC (n=48) & \\
\hline Age (yr) & $\geq 70 /<70$ & $25 / 23$ & & \\
Gender & male/female & $22 / 26$ & & \\
T & $4 b / 4 a$ & $41 / 07$ & & \\
N & N1/N0 & $24 / 24$ & & \\
M & M1/M0 & $18 / 30$ & \\
\hline
\end{tabular}

PDTC compared with low EZH2 expression (hazard ratio (HR) 9.1 ; 95\% confidence interval (CI) 1.6-50.1, $p=0.011$ ). However, high EZH2 expression was not a poor prognostic indicator of OS in ATC (HR 1.9; 95\%CI=0.7-4.9, $p=0.185$ ) (Table III), WHO PDTC (HR 4.2; 95\%CI=0.6-27.0, $p=0.137$ ), or Turin PDTC (HR 434; $95 \% \mathrm{CI}=0.0-9.0 \times 10^{12}$, $p=0.616$ ) (data not shown). High expression of EZH2 was also identified as a prognostic factor in multivariate analysis of JSTS classification PDTC (HR 9.0; 95\%CI=1.5-55.4, $p=0.018$ ), while M1 status was a poor prognostic factor in univariate and multivariate analyses of JSTS PDTC. No prognostic factors were identified for the WHO and Turin PDTC classifications. T4b and M1 status were poor prognostic factors in ATC in univariate and multivariate analyses. No factors were significantly correlated with EZH2 expression in JSTS classification PDTC, whereas tumor (T) 

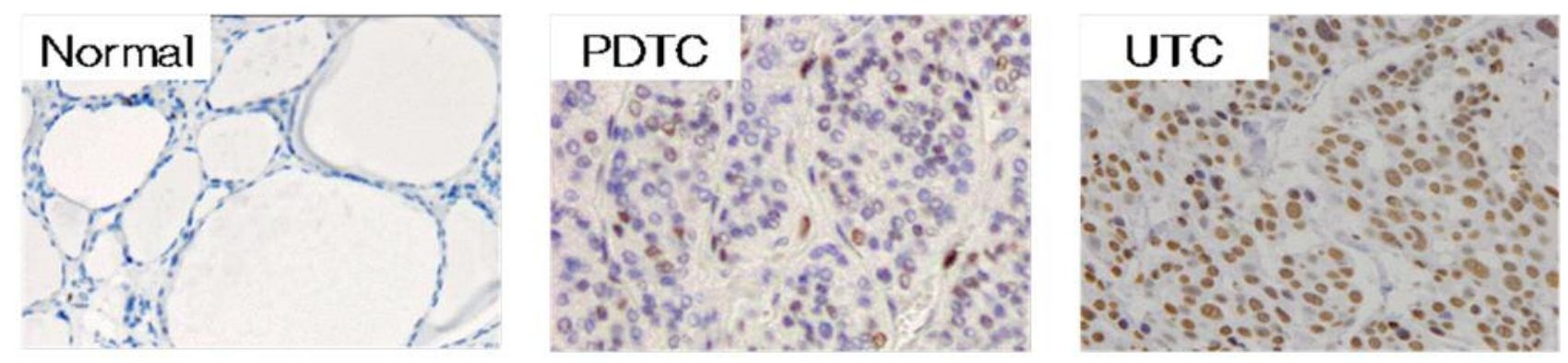

\begin{tabular}{|c|c|c|c|}
\hline \multicolumn{2}{|c|}{ Histological subtype } & Positive & Negative \\
\hline \multicolumn{2}{|c|}{ Normal } & $0.0 \%(0 / 30)$ & $100 \%(30 / 30)$ \\
\hline \multicolumn{2}{|l|}{ DTC } & $0.0 \%(0 / 30)$ & $100 \%(30 / 30)$ \\
\hline \multirow[t]{3}{*}{ PDTC } & JSTS & $17.9 \%(12 / 67)$ & $82.1 \%(55 / 67)$ \\
\hline & WHO & $21.6 \%(8 / 37)$ & $78.4 \%(29 / 37)$ \\
\hline & Turin & $25.0 \%(3 / 12)$ & $75.0 \%$ ( $9 / 12)$ \\
\hline \multicolumn{2}{|l|}{ ATC } & $87.5 \%(42 / 48)$ & $13.5 \%(6 / 48)$ \\
\hline
\end{tabular}

Figure 2. Immunohistochemical analysis of EZH2 expression in normal and neoplastic thyroid tissues. EZH2-positivity increased in the order DTC, PDTC (JSTS, WHO, Turin), and ATC.

a) All patients $(n=67)$

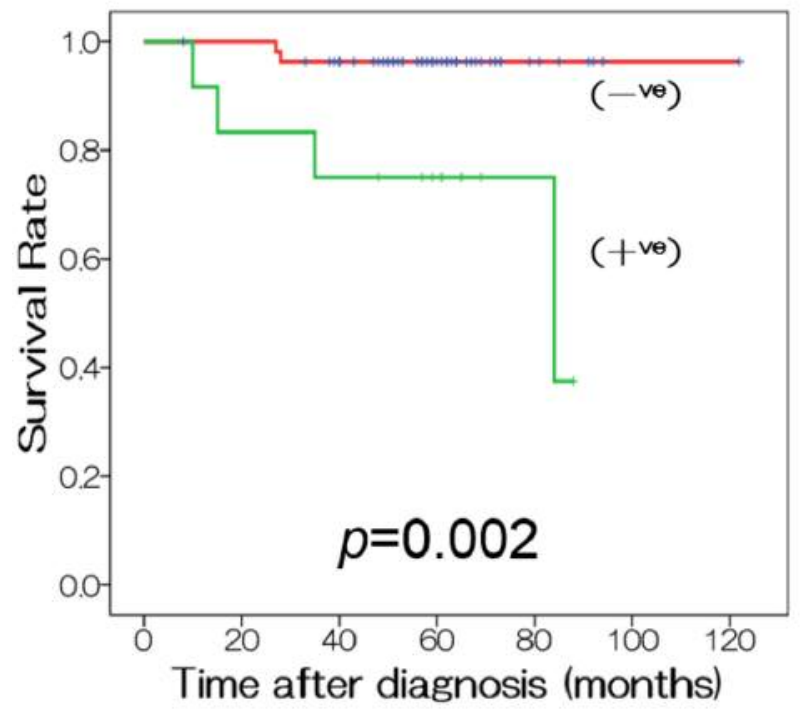

b) $\quad$ 1 patients $(n=30)$

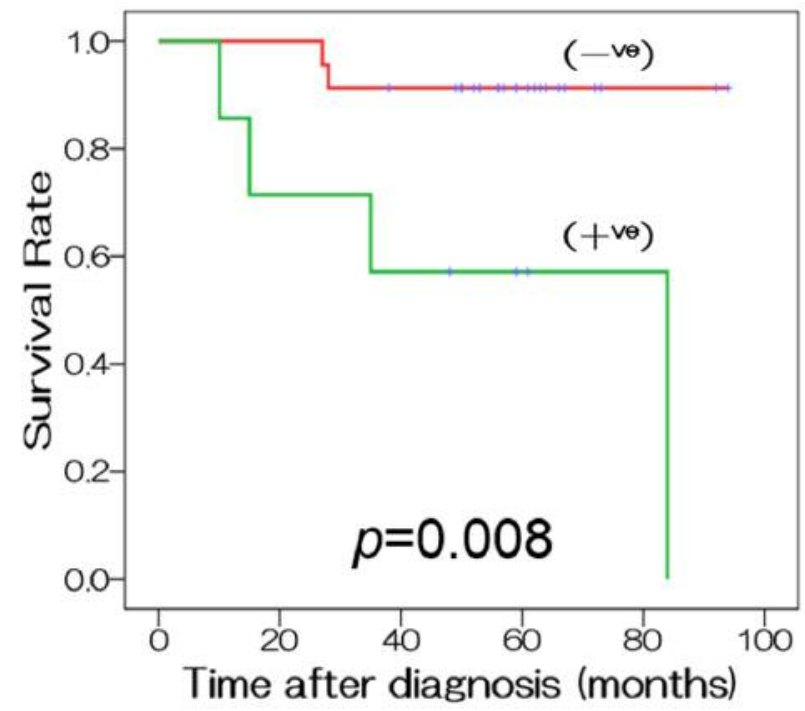

Figure 3. Kaplan-Meier survival curves for JSTS criteria PDTC with high and low EZH2 expression. The estimated 5-year survival rates in the low-expression group were significantly higher compared to the high-expression group for (a) all patients $(91.3 \% \mathrm{vs} .57 .1 \%, p=0.002)$ and $(b) \mathrm{MI}$ patients ( $83.3 \%$ vs. $40.0 \%, p=0.008)$. 
Table II. Associations between clinicopathological factors and EZH2 expression.

\begin{tabular}{|c|c|c|c|c|c|c|c|c|c|c|}
\hline & & \multicolumn{3}{|c|}{ PDTC (JSTS, n=67) } & \multicolumn{3}{|c|}{ PDTC $(\mathrm{WHO}, \mathrm{n}=37)$} & \multicolumn{3}{|c|}{ PDTC (Turin, $\mathrm{n}=12$ ) } \\
\hline & & EZH2 low & EZH2 high & $p$-Value & EZH2 low & EZH2 high & $p$-Value & EZH2 low & EZH2 high & $p$-Value \\
\hline \multirow[t]{2}{*}{ Age (yr) } & $\geq 55$ & 29 & 9 & 0.158 & 12 & 6 & 0.092 & 7 & 2 & 0.700 \\
\hline & $<55$ & 26 & 3 & & 17 & 2 & & 2 & 1 & \\
\hline \multirow[t]{2}{*}{ Gender } & male & 19 & 7 & 0.125 & 9 & 5 & 0.104 & 1 & 2 & 0.054 \\
\hline & female & 36 & 5 & & 20 & 3 & & 8 & 1 & \\
\hline \multirow[t]{2}{*}{$\mathrm{T}(\mathrm{cm})$} & $\geq 4$ & 37 & 7 & 0.738 & 25 & 4 & 0.028 & 8 & 1 & 0.054 \\
\hline & $<4$ & 18 & 5 & & 4 & 4 & & 1 & 2 & \\
\hline \multirow[t]{2}{*}{ Ex } & Ex0, 1 & 54 & 11 & 0.230 & 29 & 7 & 0.054 & 9 & 3 & 1.000 \\
\hline & Ex2 & 1 & 1 & & 0 & 1 & & 0 & 0 & \\
\hline \multirow[t]{2}{*}{$\mathrm{N}$} & No, 1a & 48 & 11 & 0.671 & 28 & 8 & 0.594 & 8 & 3 & 0.546 \\
\hline & N1b & 7 & 1 & & 1 & 0 & & 1 & 0 & \\
\hline \multirow[t]{4}{*}{ M } & M0 & 43 & 7 & 0.152 & 24 & 5 & 0.218 & 5 & 2 & 0.898 \\
\hline & M1 & 12 & 5 & & 5 & 3 & & 3 & 1 & \\
\hline & & \multicolumn{3}{|c|}{$\operatorname{ATC}(n=48)$} & & & & & & \\
\hline & & EZH2 low & EZH2 high & $p$-Value & & & & & & \\
\hline \multirow[t]{2}{*}{ Age (yr) } & $\geq 70$ & 5 & 20 & 0.101 & & & & & & \\
\hline & $<70$ & 1 & 22 & & & & & & & \\
\hline \multirow[t]{2}{*}{ Gender } & male & 1 & 21 & 0.125 & & & & & & \\
\hline & female & 5 & 21 & & & & & & & \\
\hline \multirow[t]{2}{*}{$\mathrm{T}$} & $4 a$ & 1 & 6 & 0.877 & & & & & & \\
\hline & $4 b$ & 5 & 36 & & & & & & & \\
\hline \multirow[t]{2}{*}{$\mathrm{N}$} & NO & 3 & 21 & 1.000 & & & & & & \\
\hline & $\mathrm{N} 1$ & 3 & 21 & & & & & & & \\
\hline \multirow[t]{2}{*}{ M } & M0 & 5 & 25 & 0.260 & & & & & & \\
\hline & M1 & 1 & 17 & & & & & & & \\
\hline
\end{tabular}

and extra thyroidal extension (Ex) were weakly correlated with EZH2 in ATC $(\mathrm{r}=0.379, p=0.008 ; \mathrm{r}=0.303, p=0.037$, respectively). EZH2 was an independent prognostic factor similar to $M$ status in JSTS classification PDTC. The estimated 5-year survival rates in the low-EZH2-expression group were significantly higher compared with the highexpression group in all patients $(91.3 \%$ vs. $57.1 \%, p=0.002$, Figure 3a) and in M1 patients (83.3\% vs. $40.0 \%, p=0.008)$ (Figure 3b) with JSTS PDTC.

\section{Discussion}

Three types of PDTC have been defined by the JSTS 6th edition based on the proposal by Sakamoto et al., WHO classification, and Turin proposal, respectively. Ito et al. investigated the prevalence and clinical significance of these three types (10) and confirmed that the prevalence of PDTC decreased in the order JSTS, WHO, Turin $(11.1 \%, 0.8 \%$, $0.3 \%$, respectively), while the prognosis worsened in the same order (10-year cause-specific survival: $94.2 \%, 80.0 \%$, $60.0 \%$, respectively). The current study confirmed these tendencies regarding the prevalence and prognosis. Although there was no significant difference among three types of PDTC, EZH2 protein expression tended to increase in the order JSTS (17.9\%), WHO (21.6\%), and Turin (25.0\%), in contrast to the prevalence and prognosis, while EZH2positivity increased significantly in the order DTC, PDTC, and ATC, consistent with the prognosis of thyroid cancer. The current study provides the first evidence to suggest that EZH2 expression may be an independent prognostic factor similar to metastasis, especially in JSTS PDTC.

Various possible mechanisms for EZH2 up-regulation have been revealed in different malignant tumors. The pRBE2F pathway regulates EZH2 expression by transcriptional activation and leads to tumor cell proliferation (20). In metastatic breast cancer, endothelial growth factor receptor endocytosis was suppressed, leading to EZH2 overexpression through up-regulation of the MEK-ERK-Elk-1 pathway (21), with subsequent effects of the up-regulated EZH2 on cancer proliferation. EZH2 has also been shown to repress the expression of classic tumor suppressor genes such as CDKN2A and p53 (22) directly, and to reduce the levels of 
Table III. Relationship between EZH2 expression and patient outcome.

PDTC(JSTS)

\begin{tabular}{|c|c|c|c|c|c|c|c|}
\hline \multirow[t]{2}{*}{ Factor } & \multirow[t]{2}{*}{$\mathrm{n}$} & \multicolumn{3}{|c|}{ Univariate } & \multicolumn{3}{|c|}{ Multivariate } \\
\hline & & HR & $95 \% \mathrm{CI}$ & $p$-Value & HR & $95 \% \mathrm{CI}$ & $p$-Value \\
\hline Age $(\geq 55)$ & $38 / 67(56.7 \%)$ & 2.2 & $0.4-14.0$ & 0.389 & & & \\
\hline Gender(male) & $26 / 67(38.8 \%)$ & 1.7 & $0.3-8.5$ & 0.518 & & & \\
\hline $\mathrm{T}(\geq 4 \mathrm{~cm})$ & $44 / 67(65.7 \%)$ & 5.2 & $0.5-56.8$ & 0.179 & & & \\
\hline Ex2 & $02 / 67(03.0 \%)$ & 7.7 & $0.9-69.1$ & 0.068 & 0.9 & $0.1-9.1$ & 0.954 \\
\hline $\mathrm{N} 1 \mathrm{~b}$ & $08 / 67(11.9 \%)$ & 1.2 & $0.1-10.9$ & 0.858 & & & \\
\hline M1 & $17 / 67(25.4 \%)$ & 18.4 & $2.1-159.5$ & 0.008 & 22.3 & $2.0-246.7$ & 0.011 \\
\hline $\mathrm{EZH} 2(+)$ & $12 / 67(17.9 \%)$ & 9.1 & $1.6-50.1$ & 0.011 & 9.0 & $1.5-55.4$ & 0.018 \\
\hline \multicolumn{8}{|l|}{ ATC } \\
\hline \multirow[t]{2}{*}{ Factor } & \multirow[t]{2}{*}{$\mathrm{n}$} & \multicolumn{3}{|c|}{ Univariate } & \multicolumn{3}{|c|}{ Multivariate } \\
\hline & & HR & $95 \% \mathrm{CI}$ & $p$-Value & HR & $95 \% \mathrm{CI}$ & $p$-Value \\
\hline Age $(\geq 70)$ & $25 / 48(52.0 \%)$ & 1.5 & $0.8-2.8$ & 0.166 & & & \\
\hline Gender (male) & $22 / 48(45.8 \%)$ & 0.9 & $0.8-2.8$ & 0.794 & & & \\
\hline $\mathrm{T} 4 \mathrm{~b}$ & $39 / 48(81.3 \%)$ & 2.8 & $1.2-6.3$ & 0.015 & 3.9 & $1.3-11.6$ & 0.013 \\
\hline N1 & $24 / 48(50.0 \%)$ & 1.6 & $0.9-2.8$ & 0.134 & & & \\
\hline M1 & $18 / 48(37.5 \%)$ & 2.5 & $1.3-4.6$ & 0.005 & 3.5 & $1.7-7.4$ & 0.001 \\
\hline $\mathrm{EZH} 2(+)$ & $42 / 48(87.5 \%)$ & 1.9 & $0.7-4.9$ & 0.185 & 0.9 & $0.3-2.9$ & 0.853 \\
\hline
\end{tabular}

RAD51 leading to the activation of Raf1/ERK and $\beta$-catenin signalling (23). Several reports have revealed that EZH2 affects not only genetic but also epigenetic pathways. The loss of micro RNAs such as miR-26a, miR-101, and miR214 resulted in EZH2 accumulation (24-25), while downregulation of miR-25 and miR-30d could contribute to thyroid cancer progression, leading to the development of anaplastic carcinomas targeting EZH2 mRNA (26).

Thyroid cancer is difficult to treat using conventional treatments. Some tyrosine-kinase inhibitors are currently available on the market, but new therapies are still needed Inari et al. demonstrated a significant correlation between EZH2 expression and Ki-67 expression scores in breast cancer, suggesting that EZH2 may represent a potential therapeutic target for aggressive breast cancers exhibiting high Ki-67 expression, thus warranting further investigations. Furthermore, several EZH2 inhibitors have recently been developed and have yielded promising results in small cell lung cancer cell lines and malignant rhabdoid tumors (27).

\section{Conclusion}

EZH2-positivity increases in the order of DTC, PDTC, and ATC, and high EZH2 expression appears to be a poor prognostic indicator of OS in JSTS classification PDTC.
EZH2 overexpression may be associated with the malignant potential of thyroid cancers, and may thus be a useful prognostic marker for aggressive behaviour of these cancers. EZH2 may also represent a potential therapeutic target for aggressive thyroid cancers.

\section{Conflicts of Interest}

The Authors have no conflicts of interest to declare.

\section{Acknowledgements}

This work was supported by grants from the MEXT/JSPS KAKENHI Grant Number JP 26461954. The Authors would like to express their gratitude to the staff in the Departments of Pathology, Kanagawa Cancer Centre and Ito Hospital for their technical assistance and for collecting cancer tissues. The Authors would also like to thank Susan Furness, $\mathrm{PhD}$, from Edanz Group (www.edanzediting.com/ac) for editing a draft of this manuscript.

\section{References}

1. Davies L and Welch HG: Increasing incidence of thyroid cancer in the United States, 1973-2002. JAMA 295: 2164-2167, 2006.

2 Ahn HS, Kim HJ and Welch HG: Korea's thyroid-cancer "epidemic"-screening and overdiagnosis. N Engl J Med 371: 1765-1767, 2014. 
3 Nguyen QT, Lee EJ, Huang MG, Park YI, Khullar A and Plodkowski RA: Diagnosis and treatment of patients with thyroid cancer. Am Health Drug Benefits 8: 30-40, 2015.

4 Sobrinho-Simoes M, Carcangiu ML and Albores-Saavedra J: Poorly differentiated carcinoma. In: Pathology and Genetics of Tumours of Endocrine Organs. DeLeillis RA, Lloyd RV, Heitz PU, et al. (eds.). Lyon, IARC Press, pp. 73-76, 2004.

5 Smallridge RC and Copland JA: Anaplastic thyroid carcinoma: pathogenesis and emerging therapies. Clin Oncol 6: 486-497, 2010 .

6 Sugitani I, Miyauchi A, Sugino K, Okamoto T, Yoshida A and Suzuki S: Prognostic factors and treatment outcomes for anaplastic thyroid carcinoma: ATC Research Consortium of Japan cohort study of 677 patients. World J Surg 36: 1247-1254, 2012.

7 The Japanese Society of Thyroid Surgery General Rules for the Description of Thyroid Cancer (6th edition). Tokyo, Kanehara Press (In Japanese), 2005.

8 Sakamoto A, Kasai N and Sugano H: Poorly differentiated carcinoma of the thyroid. A clinicopathologic entity for a high risk group of papillary and follicular carcinomas. Cancer 52: 1849-1855, 1983

9 Volante M, Collini P, Nikiforov YE, Sakamoto A, Kakudo K, Katoh R, Lloyd RV, LiVolsi VA, Papotti M, Sobrinho-Simoes M, Bussolati $G$ and Rosai J: Poorly differentiated thyroid carcinoma: the Turin proposal for the use of uniform diagnostic criteria and an algorithmic diagnostic approach. Am J Surg Pathol 31: 1256-1264, 2007.

10 Ito Y, Hirokawa M, Fukushima M, Inoue H, Yabuta T, Uruno T, Kihara M, Higashiyama T, Takamura Y, Miya A, Kobayashi K, Matsuzuka F and Miyauchi A: Prevalence and prognostic significance of poor differentiation and tall cell variant in papillary carcinoma in Japan. World J Surg 32: 1535-1543, 2008.

11 Cao R, Wang L, Wang H, Xia L, Erdjument-Bromage H, Tempst P, Jones RS and Zhang Y: Role of histone H3 lysine 27 methylation in Polycomb-group silencing. Science 298: 1039-1043, 2002.

12 Schuettengruber B, Chourrout D, Vervoort M, Leblanc B and Cavalli G: Genome regulation by polycomb and trithorax proteins. Cell 128: 735-745, 2007.

13 Boyer LA, Plath K, Zeitlinger J, Brambrink T, Medeiros LA, Lee TI, Levine SS, Wernig M, Tajonar A, Ray MK, Bell GW, Otte AP, Vidal M, Gifford DK, Young RA and Jaenisch R: Polycomb complexes repress developmental regulators in murine embryonic stem cells. Nature 441: 349-353, 2006.

14 Levine SS, Weiss A, Erdjument-Bromage H, Shao Z, Tempst P and Kingston RE: The core of the polycomb repressive complex is compositionally and functionally conserved in flies and humans. Mol Cell Biol 22: 6070-6078, 2002.

15 Asangani IA, Ateeq B, Cao Q, Dodson L, Pandhi M, Kunju LP, Mehra R, Lonigro RJ, Siddiqui J, Palanisamy N, Wu YM, Cao X, Kim JH, Zhao M, Qin ZS, Iyer MK, Maher CA, KumarSinha C, Varambally S and Chinnaiyan AM: Characterization of the EZH2-MMSET histone methyltransferase regulatory axis in cancer. Mol Cell 49: 80-93, 2013.

16 Kondo Y: Targeting histone methyltransferase EZH2 as cancer treatment. J Biochem 156: 249-257, 2014.

17 Borbone E, Troncone G, Ferraro A, Jasencakova Z, Stojic L, Esposito F, Hornig N, Fusco A and Orlando V: Enhancer of zeste homolog 2 overexpression has a role in the development of anaplastic thyroid carcinomas. J Clin Endocrinol Metab 96: 1029-1038, 2011.
18 Sponziello M, Durante C, Boichard A, Dima M, Puppin C, Verrienti A, Tamburrano G, Di Rocco G, Redler A, Lacroix L, Bidart JM, Schlumberger M, Damante G, Russo D and Filetti S: Epigenetic-related gene expression profile in medullary thyroid cancer revealed the overexpression of the histone methyltransferases EZH2 and SMYD3 in aggressive tumours. Mol Cell Endocrinol 392: 8-13, 2014.

19 Inari H, Suganuma N, Kawachi K, Yoshida T, Yamanaka T, Nakamura Y, Yoshihara M, Nakayama N, Yamanaka A, Masudo K, Oshima T, Yokose T, Rino Y, Shimizu S, Miyagi Y and Masuda M: Expression of enhancer of zeste homolog 2 correlates with survival outcome in patients with metastatic breast cancer: exploratory study using primary and paired metastatic lesions. BMC Cancer 17: 160-174, 2017.

20 Adrian P. Bracken AP, Pasini D, Capra M, Prosperini E, Colli E and Helin K: EZH2 is downstream of the pRB-E2F pathway, essential for proliferation and amplified in cancer. EMBO J 22: 5323-5335, 2003.

21 S Fujii, K, Tokita K, Wada N, Ito K, Yamauchi C, Ito Y and Ochiai A: MEK-ERK pathway regulates EZH2 overexpression in association with aggressive breast cancer subtypes. Oncogene 30: 4118-4128, 2011.

22 Kazanets A, Shorstova T, Hilmi K, Marques M and Witcher M: Epigenetic silencing of tumor suppressor genes: Paradigms, puzzles, and potential. Biochim Biophys Acta 1865: 275-288, 2016.

23 Yoo KH and Hennighausen L: EZH2 methyltransferase and H3K27 methylation in breast cancer. Int J Biol Sci 8: 59-65, 2012.

24 Dang X, Ma A, Yang L, Hu H, Zhu B, Shang D, Chen T and Luo Y: MicroRNA-26a regulates tumorigenic properties of EZH2 in human lung carcinoma cells. Cancer Genet 205: 113123, 2012.

25 Derfoul A, Juan AH, Difilippantonio MJ, Palanisamy N, Ried T and Sartorelli V: Decreased microRNA-214 levels in breast cancer cells coincides with increased cell proliferation, invasion and accumulation of the Polycomb Ezh2 methyltransferase. Carcinogenesis 32: 1607-1614, 2011.

26 Esposito F, Tornincasa M, Pallante P, Federico A, Borbone E, Pierantoni GM and Fusco A: Down-Regulation of the miR-25 and miR-30d contributes to the development of anaplastic thyroid carcinoma targeting the polycomb protein EZH2. J Clin Endocrinol Metab 97: E710-718, 2012.

27 Knutson SK, Warholic NM, Wigle TJ, Klaus CR, Allain CJ, Raimondi A, Porter Scott M, Chesworth R, Moyer MP, Copeland RA, Richon VM, Pollock RM, Kuntz KW and Keilhack H: Durable tumor regression in genetically altered malignant rhabdoid tumors by inhibition of methyltransferase EZH2. Proc Natl Acad Sci USA 110: 7922-7927, 2013. 